\title{
Treatment Failure with combined csDMARDs increases the risk of Low Muscle Mass in women with Rheumatoid Arthritis: a cross-sectional study
}

\section{Melissa Ramirez-Villafaña}

Unidad de Investigacion Biomedica 02, UMAE, HE, Centro Medico Nacional de Occidente, Instituto Mexicano del Seguro Social, Guadalajara, Jalisco; Programa de Doctorado en Ciencias Medicas, Universidad de Colima, Colima.

\section{N Alejandra Rodriguez-Jimenez}

Instuto de Terapeutica Experimental y Clinica, Departamento de Fisiología, Universidad de Guadalajara, Centro Universitario de Ciencias de la Salud, Guadalajara, Jalisco.

\section{Jorge I Gamez-Nava}

Unidad de Investigacion Biomedica 02, UMAE, HE, Centro Medico Nacional de Occidente, Instituto Mexicano del Seguro Social; Programa de Doctorado en Farmacologia y Programa de Doctorado en Salud Publica, Centro Universitario Ciencias de la Salud, Universidad de Guadalajara, Guadalajara, Jalisco.

\section{Javier A Aceves-Aceves}

Programa de Doctorado en Farmacologia, Universidad de Guadalajara Centro Universitario de Ciencias de la Salud, Guadalajara, Jalisco.

\section{Ana M Saldaña-Cruz}

Instituto de Terapeutica Experimental y Clinica, Departamento de Fisiologia, Universidad de Guadalajara, Centro Universitario de Ciencias de la Salud, Guadalajara, Jalisco

\section{Ernesto G. Cardona-Muñoz}

Departamento de Fisiologia, Universidad de Guadalajara, Centro Universitario de Ciencias de la Salud, Guadalajara, Jalisco

\section{Sylvia E Totsuka-Sutto}

Departamento de Fisiologia, Universidad de Guadalajara, Centro Universitario de Ciencias de la Salud, Guadalajara, Jalisco.

\section{J Manuel Ponce-Guarneros}

Departamento de Fisiologia, Universidad de Guadalajara, Centro Universitario de Ciencias de la Salud, Guadalajara, Jalisco.

\section{Fidencio Cons-Molina}

Centro de Investigacion de Artritis y Osteoporosis, Mexicali, Baja California.

\section{Xochitl Trujillo}

Universidad de Colima, Centro Universitario de Investigaciones Biomedicas, Colima, Colima. 


\section{Miguel Huerta}

Universidad de Colima, Centro Universitario de Investigaciones Biomedicas, Colima, Colima.

\section{Alfonso J Cruz-Jentoft}

Servicio de Geriatria, Hospital Universitario Ramon y Cajal (IRYCIS), Madrid.

Laura Gonzalez-Lopez ( $\sim$ dralauragonzalez@prodigy.net.mx )

Universidad de Guadalajara, Centro Universitario Ciencias de la Salud https://orcid.org/0000-00026517-6344

\section{Research article}

Keywords: rheumatoid arthritis, low skeletal muscle, therapy failure, csDMARDs

Posted Date: May 1st, 2020

DOI: https://doi.org/10.21203/rs.3.rs-25713/v1

License: (c) (1) This work is licensed under a Creative Commons Attribution 4.0 International License.

Read Full License 


\section{Abstract}

\section{Background}

Low muscle mass (myopenia) is frequent in rheumatoid arthritis (RA) patients with a long-disease duration. Although, the use of combined therapy with conventional synthetic DMARDs (csDMARDs) is one of the main strategies observed in these patients; there is a lack of information if the failure to these therapies increases the risk of myopenia. Objective: To evaluate whether the treatment failure to combined therapy with conventional synthetic DMARDs is an independent risk factor for low skeletal muscle mass in women with RA.

Methods

This cross-sectional study compared 277 women with RA (cases) and 237 women from non-rheumatic population (controls). In RA patients, we assessed clinical, epidemiological, and therapeutic variables (identifying treatment failure to combined therapy with csDMARDs. The skeletal muscle index (SMI) was estimated by DXA. Low skeletal muscle mass was defined as SMl $5.45 \mathrm{~kg} / \mathrm{m} 2$. Multivariate logistic regression analyses were used to a) evaluate whether RA is an independent risk factor of myopenia in comparison with non-rheumatic population and b) identify if treatment failure with csDMARDs is an independent risk factor of myopenia in RA.

Results

RA patients had a higher prevalence of low skeletal muscle mass than controls ( $27.8 \%$ vs. $15.6 \%$, $p=0.001)$. After adjusting for other factors, RA patients had higher risk of low skeletal muscle mass than controls (OR: 2.7, $95 \% \mathrm{Cl}: 1.7$ to 4.5). Risk factors of low muscle mass in RA patients; were: menopause (OR: $2.3,95 \% \mathrm{Cl}: 1.2$ to $4.6, \mathrm{p}=0.02$ ) and a failure to combined therapy with csDMARDs (OR: $2.4,95 \% \mathrm{Cl}$ : 1.10 to $5.81, p=0.03)$.

Conclusions

Rheumatoid arthritis is associated with an increased risk of myopenia. Treatment Failure with conventional-synthetic DMARDs constitutes a strong risk factor for deteriorated skeletal muscle mass.

\section{Introduction}

A low skeletal muscle mass (also known as myopenia) is now listed in the diagnostic criteria of many conditions, including malnutrition, cachexia and sarcopenia (1-3). Patients with inflammatory rheumatic diseases have usually, a higher frequency of low skeletal muscle mass than non-rheumatic population. In rheumatoid arthritis (RA) patients, the presence of a low skeletal muscle mass has been associated in several studies with sarcopenia or cachexia $(4,5)$. 
Although the majority of the studies performed in RA patients conclude that the frequency of low skeletal muscle mass is higher than that observed in controls (6-10), the prevalence rates of myopenia in RA vary widely from $11 \%$ to $49 \%(11,12)$. A deteriorated skeletal muscle mass should be detected early and treated adequately in RA patients because low muscle mass may be associated with muscle weakness, impairment of functioning, and deteriorated health-related quality of life, among other adverse consequences $(13,14,5)$.

The factors associated with low muscle mass in RA include the persistence of active disease with moderate to severe inflammation accomplished of increased acute phase reactants levels (12-14). With these data, it can be hypothesized that a failure to achieve an adequate therapeutic response and persistence of an active disease could be a major risk factor for deteriorated muscle mass. Nevertheless, to date, there is only limited and inconsistent information about the relation between low skeletal muscle mass and the type of drugs used for the treatment in RA $(15,12,6,8,10,16)$. Treatment with conventional synthetic disease-modifying antirheumatic drugs (cSDMARDs) is considered the first line of treatment in RA patients and should be prescribed early $(17,18)$. Combination therapy with csDMARDs is an adequate therapeutic strategy after the failure to monotherapy, and the combination of csDMARDs has demonstrated higher effectivity than monotherapy with a DMARD $(17,19-21)$. In developing countries where many RA patients have economic restrictions difficulting subsequent therapy with biologic agents, the use of combined therapy with csDMARDs constitutes a frequent therapeutic strategy $(22,23)$. Failure to combined therapy with csDMARDs is associated with worsening of the disease, radiological damage progression and other negative consequences for the patients, including a high risk of permanent work disability $(24,25)$.

To date, there is a lack of information on whether the failure to combined therapy with csDMARDs is associated with myopenia in patients with RA. Therefore, this study evaluated whether the failure to combined csDMARDs is an independent risk factor for low skeletal muscle mass in women with RA.

\section{Methods}

Objective: To evaluate whether the treatment failure to combined therapy with conventional synthetic DMARDs is an independent risk factor for low skeletal muscle mass in women with RA.

This cross-sectional study was performed in 277 Mexican women with RA who were recruited by invitation in an outpatient research clinic of chronic diseases at the University of Guadalajara (Instituto de Terapeutica Experimental y Clinica (INTEC), Centro Universitario de Ciencias de la Salud, Universidad de Guadalajara) in Guadalajara city, Mexico. Patients were eligible to participate if they met the 1987 American College of Rheumatology (ACR) criteria for RA (26), withaged $\geq 18$ years, and signed a voluntary consent form for participation prior to their inclusion.

We excluded overlapping syndromes, infections (including acute infections, chronic viral infections such as hepatitis $\mathrm{B}$ or $\mathrm{C}$, human immunodeficiency virus and tuberculosis). Patients who were pregnant or breastfeeding were also excluded. Other exclusion criteria were active cancer, hypothyroidism, chronic 
renal failure (defined as a serum creatinine $>1.2 \mathrm{mg} / \mathrm{dL}$ and an estimated glomerular filtration rate lower than $50 \mathrm{~mL} /$ minute), and an increase in transaminase levels to greater than 2 -fold of normal values according to our laboratory.

We invited a group of women $(n=237)$ of a similar range of age ( \pm 3 years) regarding RA patients to participate as controls. These controls were patients attending the same outpatient research clinic in Guadalajara city, Mexico. The reasons for the consultation of these patients included as controls were any of the following diagnoses: hypertension, obesity, diabetes mellitus, dyslipidemia, osteoporosis or metabolic syndrome. All the patients invited to participate as controls in the study signed an informed consent form. We excluded those potential controls if they had antecedents of any inflammatory autoimmune disorders or if they had any of the exclusion criteria described above for RA patients.

\section{Study Protocol}

RA patients and controls were interviewed and clinically examined by trained researchers. These researchers performed a chart review assessing epidemiological and clinical characteristics. Bodyweight was determined using a calibrated weighting machine. Height was measured using a wall stadiometer. Body mass index (BMl) was calculated in $\mathrm{kg} / \mathrm{m}^{2}$ and classified into the following categories: low weight $\left(<18.5 \mathrm{~kg} / \mathrm{m}^{2}\right)$, normal weight (range from $18.5-24.9 \mathrm{~kg} / \mathrm{m}^{2}$ ), overweight (ranging from $25-29.9 \mathrm{~kg} / \mathrm{m}^{2}$ ), and obesity $\left(\geq 30 \mathrm{~kg} / \mathrm{m}^{2}\right)(27)$.

\section{Clinical Assessment of Patients with Rheumatoid Arthritis}

DAS28-ESR index, was used to classify RA patients into two groups: a) active disease, (DAS28-ESR $\geq 2.6$ ); and $b$ ) inactive disease (DAS28-ESR score of $<2.6)(28,29)$. Physical functioning was investigated with the validated Spanish version of the Health Assessment Questionnaire-Disability index (HAQ-Di) (30).

\section{Body Composition Measurements}

Body composition was assessed by dual-energy X-ray absorptiometry (DXA) (LUNAR 2000, Prodigy Advance; General Electric ${ }^{\mathrm{TM}}$, Madison, WI, USA). All the studies were performed by trained researchers in DXA studies (following the guides of the International Society for Clinical Densitometry (ISCD)(31, 32). The following measures were estimated: fat mass (\%); lean mass: total and in appendicular legs and arms (gr \%); and total bone mineral density (BMD) $\left(\mathrm{gr} / \mathrm{cm}^{2}\right)$.

Muscle mass was estimated by DXA, computing the skeletal muscle index (SMI) according to the validated protocol described by Baumgartner et al. (20). To calculate SMI, the skeletal muscle mass of the extremities (SMME) was first computed as the sum of the muscle mass of the four limbs obtained from DXA results, and then SMME was adjusted by height (meters squared) (SMI = SMME $/$ height $\left.^{2}\right)(12)$. 
The result of this computation gives a value of SMI expressed in $\mathrm{kg} / \mathrm{m}^{2}$. According to Baumgartner et al., $\mathrm{SMI}$ is considered low in women when the result obtained is $<5.45 \mathrm{~kg} / \mathrm{m}^{2}$ independently of age (33).

\section{Pharmacological Treatment}

In our clinical setting, once patients are diagnosed with RA by a rheumatologist, the first step is the prescription of monotherapy with a csDMARDs as the first line of treatment. The csDMARDs most commonly used in monotherapy is methotrexate unless there is a contraindication for this drug; in that case, other csDMARDs such as sulfasalazine, antimalarials, leflunomide or azathioprine are initiated based on patient preferences and the clinical judgement of the rheumatologist. After three to fourth months of monotherapy with the initial csDMARDs, patients are re-assessed, and according to the rheumatologist's assessment of achieving a therapeutic response and the presence or absence of side effects, patients may be recommended by their rheumatologists to continue with the initial csDMARDs, instead if a satisfactory response is not achieved then the rheumatologists can use one of the following strategies a) change to a second csDMARDs as monotherapy, c) start a biologic DMARD (this strategy is less frequently used by economic issues), or d) to use the step-up strategy of combined therapy with two or more csDMARDs. The step-up approach with csDMARDs is the therapeutic strategy most commonly used in our setting after failure to monotherapy with a csDMARDs. The use of biologic DMARDs is planned considering economic restrictions, taking into account the patients' decision and economic feasibility.

Definition of failure to combined therapy with csDMARDs:

For patients who started a combined therapy with csDMARDs (unless side effects developed), this therapy was maintained for at least 3 to 4 months using recommended doses. A first failure to combined therapy with csDMARDs was considered following the EULAR criteria of poor response if the patients remained with a DAS28-PCR >3.2 (34). According the treat-to target guidelines these patients did not achieved remission or low-disease activity (35). In RA patients with a first failure to combined therapy, a second approach using different combination of csDMARDs was used. Definite failure to combined therapy with csDMARDs was considered when the patients did not achieve remission or at least lowdisease activity with a second different combined therapy with csDMARDs.

\section{Laboratory Determinations of Rheumatoid Factor, Acute Phase Reactants, Anti-CCP, and Other Biomarkers}

After an eight-hour fast, a sample of peripheral venous blood was obtained on the same day of the assessment from each patient in both groups (RA and controls). Serum was stored at $-20^{\circ} \mathrm{C}$. Serum levels of tumor necrosis factor-a (TNF-a), interleukin 6 (IL-6), and Anti-Cyclic Citrullined Peptide (AntiCCP) antibodies were measured by ELISA using commercial kits (R\&D Systems ${ }^{\text {TM }}$, Minneapolis, MN, USA for TNF-a, and Euroimmun AG ${ }^{T M}$, Luebeck, Germany for Anti-CCP). Rheumatoid factor (RF) was measured 
by nephelometry, erythrocyte sedimentation rate (ESR) was measured by Wintrobe, and C-reactive protein (PCR) was quantified by nephelometry.

\section{Statistical Analysis}

Quantitative variables were described as the means and standard deviations (SD), and qualitative variables were described as frequencies and (\%). Comparisons of proportions between groups were made using the Chi-square test (or Fisher exact test, when required), whereas comparisons of means between two groups were computed using independent-samples Student's $t$ test. We performed multivariate logistic regression analyses to identify risk factors of low skeletal muscle mass (dependent variable) while adjusting for potential confounders. Covariates introduced in the models were variables with a $p$ value $\leq 0.20$ in the univariate analyses or those considered with biological plausibility for influencing this dependent variable. The models were also adjusted by age and disease duration. We utilized the forward stepwise method. Statistical significance was set at the $p \leq 0.05$ level. All statistical analyses were performed using SPSS statistical software for Windows version 25.0.

\section{Results}

A total of 514 women were included, 277 of whom had RA compared with 237 were controls. Table 1 shows the clinical characteristics of the RA patients. The mean age was 57.1 years, and the disease duration was 10.7 years. Of 277 RA patients, $94.2 \%$ had taken the prescribed treatment with synthetic DMARDS (5.8\% were not compliant with the prescription), and $10.5 \%$ had repeated failure to combined therapy with csDMARDs and were programed to starting to be treated with a biologic-DMARD (Table 1).

Table 2 shows the comparison of epidemiological characteristics and comorbidities between RA patients and controls. Patients with RA had higher rates of having a sedentary lifestyle, hormone replacement therapy, dyslipidemia and osteoporosis. RA patients also had a lower frequency of alcohol consumption, diabetes mellitus, or metabolic syndrome. Mean skeletal muscle Index (SMI) was lower in the RA group than in the CS group (6.031.1 vs 6.330.93). Remarkably, RA patients had a higher prevalence of low skeletal muscle mass $(\mathrm{SMl}<5.45 \mathrm{~kg} / \mathrm{m} 2)$ than the non-rheumatic population $(27.8 \%$ vs $15.6 \%, \mathrm{p}=0.001)$. Because of the differences in the prevalence of risk factors of low muscular mass, we performed a subsequent adjusted analysis including age, alcohol consumption, sedentary lifestyle, menopause, hypertension, diabetes mellitus and metabolic syndrome as confounders (see table 4).

Table 3 compares the clinical, anthropometric and lifestyle characteristics in RA patients with and without low skeletal muscle mass. RA + low skeletal muscle mass had lower weight, lower frequency of menopause and higher frequency of treatment failure to combined synthetic DMARDs.

RA + low skeletal muscle mass also had a non-significant trend for higher doses of corticosteroids $(p=$ $0.09)$, higher CRP levels $(p=0.06)$ and lower prevalence of diabetes $(p=0.06)$. We did not observe significant differences on other variables in the univariate analysis. 
Table 4 shows the results of the logistic regression analysis, assessing the variables associated with low skeletal muscle mass in RA patients and controls (Model 1) or assessing low skeletal muscle mass in RA patients exclusively (Model 2). In Model 1, $\mathrm{n}=514$ ) after adjustment for age, alcohol consumption, sedentary lifestyle, menopause, and comorbidities such as hypertension, diabetes mellitus and metabolic syndrome, the presence of RA increased the risk of low skeletal muscle mass almost 3-fold compared to the risk observed in controls (OR: $2.7,95 \% \mathrm{Cl}: 1.68$ to 4.5 ). A protective factor for low skeletal muscle mass was higher BMI (OR: $0.15,95 \% \mathrm{Cl}: 0.09$ to 0.23 ). In the multivariable model number 2 performed in 277 RA patients (table 5), after adjustment for age, smoking, disease duration, corticosteroid dosage, disease activity, HAQ-DI score, and comorbidities. The main risk factors of low skeletal muscle mass in RA were menopause (OR: $2.3,95 \% \mathrm{Cl}: 1.16$ to 4.58 ) and failure to combined therapy with synthetic DMARDs (OR: $2.4,95 \% \mathrm{Cl}: 1.09$ to 5.37 ).

\section{Discussion}

In the present study, we found that, after controlling for confounders, RA increases the risk of having a low skeletal muscle mass in women almost 3-fold compared with that in female controls of similar age. After adjusting for age, smoking, disease duration, corticosteroid dosage, disease activity, HAQ-DI score, $\mathrm{BMI}$ and comorbidities, we also found that a failure to combined treatment with csDMARDs was associated with a 2.4 -fold risk of low skeletal muscle mass.

Torii et al. (12) described a higher prevalence of low muscle mass in RA patients (49\%) in Japan, and similar findings were also found by two independent studies performed in RA patients from Morocco and Turkey $(39.8 \%$ and $43.3 \%$, respectively) $(13,8)$. However, the rate of low skeletal muscle mass observed in our patients with RA was lower (27.8\%), closer to that found in studies performed in Europe and in the United States, in which the reported rates of low muscle mass vary from $20 \%$ to $28.6 \%(7,10)$. An even lower prevalence of low muscle mass in RA has been reported in two additional studies, one of which was performed in Spain and the second of which was reported in the United States (13\% and $11 \%$, respectively) $(9,11)$. Such variations can be attributable to differences in the population of RA patients being included, such as differences in race and genetic predisposition, different RA duration at the time of the study, more severe disease at the time of the inclusion, contribution of comorbid diseases and nonsimilarity of the therapeutic strategies. Also, these studies have a high heterogeneity in their inclusion criteria $(6,9,13,12)$.

This issue is important because there are several variables that can influence the presence of myopenia, including sex, age, severity of the disease and comorbidities, which should be considered in comparing the results across the different studies.

Our findings of a higher prevalence of low muscle mass in women with RA compared with controls are consistent with the data reported by other case-control studies. A similar result was identified by Alkan et al. in Turkey with a rate of low muscle mass of $20 \%$ in their patients with RA versus $7 \%$ in their controls (7). 
Several studies have also compared the frequency of low skeletal muscle mass in RA patients with controls $(6,8-10)$. Delgado-Frias et al. in Spain described a frequency of low muscle mass of $13 \%$ in RA patients compared to $6 \%$ in controls (9), whereas Tournadre et al. in France identified a frequency of low muscle mass of $28.6 \%$ in RA patients but only in $4.8 \%$ in their controls (10). These studies support our findings that the presence of RA increases the risk of low muscle mass in comparison with the population included as a control.

Nevertheless, relatively few studies have identified the risk of low muscle mass in RA adjusting by confounders $(6,9,10,12,13)$. After controlling the multivariate logistic regression analysis for other potential risk factors, we identified that the risk of low skeletal muscle mass is increased in RA patients, independently of the other traditional factors associated with sarcopenia. This fact should alert physicians to detect this feature earlier in RA patients and establish adequate strategies for avoiding complications.

In relation to the main aim of our study, we demonstrated a significant association between the failure to combined therapy with csDMARDs and low muscle skeletal mass in RA. To our best knowledge, this finding has not been reported previously. Other works have tested the link between treatment with glucocorticoids and myopenia, an association that was not observed in our study, probably because our patients used lower corticosteroid doses. Ngeleu et al., for example, reported in their RA patients a higher dose of glucocorticoids compared with our study (mean $11 \mathrm{mg} /$ day vs. $4.9 \mathrm{mg} /$ day, respectively), with corticosteroids being a drug that in high doses contributes to the decrease in skeletal muscle mass.

Dao et al. explored the association between low muscle mass and the lack of treatment with DMARDs, identifying a higher frequency of low muscle mass and obesity (sarcopenic obesity) in patients with RA not being treated with synthetic disease modifying anti-rheumatic drugs (DMARDs) (16).

The results of this work support our hypothesisthat in RA patients, the failure to achieve adequate control of the disease is a risk factor for impaired muscle mass. This is consistent with the knowledge that persistent inflammatory disease activity, a decrease in physical performance, and the overexpression of pro-inflammatory cytokines, adipokines and other molecules increase muscle degradation, leading to the development of low muscle mass in these patients $(5,14)$.Inflammation has been shown to reduce muscle mass in both cachexia and sarcopenia. We consider our findings that a failure to combined treatment with csDMARDs is associated with a greater risk of low skeletal muscle mass in RA women to be relevant for clinical care in RA patients. Therefore, other therapeutic strategies might need to be considered earlier in non-responders to combined therapy with csDMARDS. Some studies have identified that biological agents such as etanercept or tocilizumab could have a protective effect on the maintenance of adequate lean mass $(10,15,36)$. Due to their potent effects in suppressing inflammation by inhibiting cytokines such as TNF-a and IL-6, biologic agents might reduce the catabolic effects of these and other molecules, thus collaborating with the positive effects on muscle associated with a control of the disease activity $(10,37)$. 
An evident limitation of our study is the late start of biological therapies, which are usually not considered until two or three different combinations of csDMARDs fail, so we did not have a group with early biological treatment for comparison. However, we used a therapeutic protocol that is common in countries with financial restrictions on the use of expensive drugs, and thus our findings may be useful for many clinical settings. A future cohort comparing RA patients treated early with biologics versus treated with combined therapy with csDMARDs at a similar point of disease duration is required.

Other limitations of our study should be noted. We did not measure nutritional status, and malnutrition is a precipitant factor for muscle deterioration. Future studies should include the analysis of nutritional status as a relevant factor in these patients with RA. Also, this study focused in the measurement of muscle mass, but a complete assessment of muscle function, muscular strength, and assessment of nutrition in conjunction with muscle mass is relevant for a more complete assessment of sarcopenia (5, 3 ). Rheumatoid cachexia instead is a syndrome characterized by a loss of cell mass and body mass not only involving skeletal muscle but also usually involving a decrease in fat mass $(4,5)$. A complete assessment of sarcopenia, rheumatoid cachexia, and malnutrition evaluation of these three features should be considered in future studies.

\section{Conclusions}

RA increases the risk of having a low skeletal muscle mass in Mexican women by $>2$-fold compared with women of similar age. Continued failure to combined csDMARDs is a strong risk factor for low skeletal muscle mass in RA, independent of traditional risk factors. An early detection of therapy failure to combined csDMARDs should be followed by considering other treatments and measures could contribute to avoiding the negative effects of this therapy failure on low skeletal muscle mass in RA patients and their consequences. Further long-term studies and follow-up are required to identify the most valuable measures to improve the loss of muscle mass, thus decreasing the impact of low skeletal muscle mass in these patients with RA.

\section{List Of Abbreviations}

ACR:American College of Rheumatology, Anti-CCP: antibodies against a cyclic citrullinated peptide, BMD: bone mineral density, BMl: Body mass Index, csDMARDs: conventional synthetic-Disease-Modifying AntiRheumatic Drugs, CRP: C-reactive protein, DAS28-ESR: Disease Activity Score for 28 joints with Erythrocyte Sedimentation Rate, DMARDs: Disease-Modifying Anti-Rheumatic Drugs, DXA: Dual-energy Xray Absorptiometry, ESR: Erythrocyte sedimentation rate HAD-Di:Health Assessment Questionnaire, IL-6: Interleukine-6, INTEC: Instituto de Terapeutica Experimental y Clinica, ISCD: the International Society for Clinical Densitometry, RA:Rheumatoid Arthritis, RF: Rheumatoid Factor, SD: standard deviations, SMI: skeletal muscle index, SMME: skeletal muscle mass of the extremities, TNF-a:Tumor necrosis factoralpha

\section{Declarations}




\section{Ethics}

The study protocol was performed following the lineaments of the Helsinki Declaration. Prior to participation, all the participants signed a voluntary informed consent form. All the procedures involved in this study had the approval of the Research and Ethics Committee of the Instituto de Terapeutica Experimental y Clinica, Centro Universitario de Ciencias de la Salud, Universidad de Guadalajara. Numero de Aprobacion (Code of approval): CEI/499/2019

\section{Consent for publication}

"Not applicable"

\section{Availability of data and materials:}

The dataset supporting the conclusions presented in this article is available on request from the corresponding author.

\section{Competing Interest}

All the authors have read and approved the final version of the manuscript that we are submitting and declare that there is no conflict of interests regarding to the publication of this article.

\section{Funding}

No specific funding was received from any bodies in the public, commercial or not-for-profit sectors to carry out the work described in this article

\section{Author's contributions:}

Conceptualization: L G-L, JI G-N, AJ C-J, EG C-M, F C-M, NA R-J and M R-V; Methodology: L G-L, AJ C-J, AM S-C, X T, M H, S T-S.; Project administration: EG C-M; SE T-S; Formal Analysis: JI G-N, L G-L and JA A-A; Investigation: M R-V, NA R-J, AM S-C, JM P-G, JA A-A; Resources: L G-L, EG C-M, M H, X T; Writing Original Draft Preparation: M R-V, NA R-J, JA A-A, M H, X T, AJ C-J, JI G-N and L G-L; Writing - Review \& Editing: JI G-N, L G-L, AJ C-J, F C-M; Visualization: M R-V, NA R-J, JA A-A, JM P-G, AJ C-J, JI G-N and L G-L; Supervision: AJ C-J, F C-M, and L G-L; Project Administration: L G-L and EG C-M. Approbation of the final version of this manuscript: M R-V, NA R, JI G-N, JA A-A, AM S-C, EG C-M, SE T-S, JM P-G, F C-M,X T, M H, AJ C-J, L G-L.

\section{Acknowledgements}

Dr. Laura Gonzalez-Lopez holds the Beca de Excelencia en Investigación 2016-2018 by the Fundación IMSS, A. C. (Foundation IMSS, A. C research scholarship 2016 - 2018). PhD Melissa Ramírez-Villafaña is the recipient of funding for postgraduate studies provided by a Consejo Nacional de Ciencia y Tecnología (Mexican Council of Science and Technology CONACyT) PhD scholarship. 


\section{References}

1. Cederholm T, Jensen GL, Correia MITD, Gonzalez MC, Fukushima R, Higashiguchi T, et al; GLIM Core Leadership Committee; GLIM Working Group. GLIM criteria for the diagnosis of malnutrition - A consensus report from the global clinical nutrition community. Clin Nutr. 2019; 38:1.

2. Muscaritoli M, Anker SD, Argilés J, Aversa Z, Bauer JM, Biolo G, et al. Consensus definition of sarcopenia, cachexia and pre-cachexia: joint document elaborated by Special Interest Groups (SIG) "cachexia-anorexia in chronic wasting diseases" and "nutrition in geriatrics". Clin Nutr. 2010; 29:1549.

3. Cruz-Jentoft AJ, Baeyens JP, Bauer JM, Boirie Y, Cederholm T, Landi F, et al. European Working Group on Sarcopenia in Older People. Age Ageing. Sarcopenia: European consensus on definition and diagnosis: Report of the European Working Group on Sarcopenia in Older People. Age Ageing. 2010; 39:412-23.

4. El Maghraoui A, Sadni S, Rezqi A, Bezza A, Achemlal L, Mounach A. Does Rheumatoid Cachexia Predispose Patients with Rheumatoid Arthritis to Osteoporosis and Vertebral Fractures? J Rheumatol. 2015; 42:1556-62.

5. Walsmith J, Roubenoff R. Cachexia in rheumatoid arthritis. Int J Cardiol. 2002; 85:89-99.

6. Giles JT, Ling SM, Ferrucci L, Bartlett SJ, Andersen RE, Towns M, et al. Abnormal body composition phenotypes in older rheumatoid arthritis patients: association with disease characteristics and pharmacotherapies. Arthritis Rheum. 2008; 59: 807-15.

7. Alkan Melikoğlu M. Presarcopenia and its Impact on Disability in Female Patients With Rheumatoid Arthritis. Arch Rheumatol. 2017; 32:53-59.

8. Doğan SC, Hizmetli S, Hayta E, Kaptanoğlu E, Erselcan T, Güler E. Sarcopenia in women with rheumatoid arthritis. Eur J Rheumatol. 2015; 2:57-61.

9. Delgado-Frías E, González-Gay MA, Muñiz-Montes JR, Gómez Rodríguez-Bethencourt MA, GonzálezDíaz A, Díaz-González F, et al. Relationship of abdominal adiposity and body composition with endothelial dysfunction in patients with rheumatoid arthritis. Clin Exp Rheumatol. 2015; 33:516-23.

10. Tournadre A, Pereira B, Dutheil F, Giraud C, Courteix D, Sapin V, et al. Changes in body composition and metabolic profile during interleukin 6 inhibition in rheumatoid arthritis. $J$ Cachexia Sarcopenia Muscle. 2017; 8: 639-46.

11. Baker JF, Long J, Ibrahim S, Leonard MB, Katz P. Are men at greater risk of lean mass deficits in rheumatoid arthritis? Arthritis Care Res (Hoboken). 2015; 67:112-9.

12. Torii $M$, Hashimoto $M$, Hanai A, Fujii T, Furu M, Ito $H$, et al, Prevalence and factors associated with sarcopenia in patients with rheumatoid arthritis. Mod Rheumatol. 2019;29: 589-595.

13. Ngeuleu A, Allali F, Medrare L, Madhi A, Rkain H, Hajjaj-Hassouni N. Sarcopenia in rheumatoid arthritis: prevalence, influence of disease activity and associated factors. Rheumatol Int. 2017; 37:1015-20. 
14. Giles JT, Bartlett SJ, Andersen RE, Fontaine KR, Bathon JM. Association of body composition with disability in rheumatoid arthritis: impact of appendicular fat and lean tissue mass. Arthritis Care Res. 2008; 59:1407-115.

15. Marcora SM, Chester KR, Mittal G, Lemmey AB, Maddison PJ. Randomized phase 2 trial of anti-tumor necrosis factor therapy for cachexia in patients with early rheumatoid arthritis. Am J Clin Nutr. 2006: 84:1463-72

16. Dao HH, Do QT, Sakamoto J. Abnormal body composition phenotypes in Vietnamese women with early rheumatoid arthritis. Rheumatology (Oxford). 2011; 50:1250-8.

17. Smolen JS, Landewé R, Bijlsma J, Burmester G, Chatzidionysiou K, Dougados M, et al. EULAR recommendations for the management of rheumatoid arthritis with synthetic and biological diseasemodifying antirheumatic drugs:2016 update. Ann Rheum Dis. 2017;76: 960-977.

18. Singh JA, Furst DE, Bharat A, Curtis JR, Kavanaugh AF, Kremer JM, et.al. 2012 update of the 2008 American College of Rheumatology recommendations for the use of disease-modifying antirheumatic drugs and biologic agents in the treatment of rheumatoid arthritis. Arthritis Care Res (Hoboken). 2012; 64:625-39.

19. De Jong PH, Hazes JM, Barendregt PJ, Huisman M, van Zeben D, van der Lubbe PA, et al. Induction therapy with a combination of DMARDs is better than methotrexate monotherapy: first results of the tREACH trial. Ann Rheum Dis. 2013; 72:72-8.

20. Van Tuyl LHD, Plass AMC, Lems WF, Voskuyl AE, Kerstens PJ, Dijkmans BA, et al. Discordant perspectives of rheumatologists and patients on COBRA combination therapy in rheumatoid arthritis. Rheumatology (Oxford). 2008; 47:1571-1576.

21. Boers M, Verhoeven AC, Markusse HM, Van De Laar MA, Westhovens R, Van Denderen JC, Randomised comparison of combined step-down prednisolone, methotrexate and sulphasalazine with sulphasalazine alone in early rheumatoid arthritis. Lancet. 1997; 350:309-318.

22. Mittal N, Mittal R, Sharma A, Jose V, Wanchu A, Singh S. Treatment failure with disease-modifying antirheumatic drugs in rheumatoid arthritis patients. Singapore Med J. 2012; 53:532-6.

23. Goycochea-Robles MV, Arce-Salinas CA, Guzmán-Vázquez S, Cardiel-Ríos MH. Prescription rheumatology practices among Mexican specialists. Arch Med Res. 2007; 38: 354-9.

24. van der Kooij SM, de Vries-Bouwstra JK, Goekoop-Ruiterman YP, van Zeben D, Kerstens PJ, Gerards $\mathrm{AH}$, et al. Limited efficacy of conventional DMARDs after initial methotrexate failure in patients with recent onset rheumatoid arthritis treated according to the disease activity score. Ann Rheum Dis. 2007; 66:1356-62.

25. Vazquez-Villegas ML, Gamez-Nava JI, Celis A, Sanchez-Mosco D, de la Cerda-Trujillo LF, MurilloVazquez JD, et al. Prognostic Factors for Permanent Work Disability in Patients with Rheumatoid Arthritis Who Received Combination Therapy of Conventional Synthetic Disease-Modifying Antirheumatic Drugs: A Retrospective Cohort Study. J Clin Rheumatol. 2017; 23:376-382.

26. Arnett FC, Edworthy SM, Bloch DA, McShane DJ, Fries JF, Cooper NS, et al. The American Rheumatism Association 1987 Revised Criteria for the Classification of Rheumatoid Arthritis. 
Arthritis Rheum. 1988; 31: 315-24.

27. WHO Consultation on Obesity (1999: Geneva, Switzerland) \& World Health Organization. (2000). Obesity: preventing and managing the global epidemic report of a WHO consultation. Geneva: World Health Organization. http://www.who.int/iris/handle/10665/42330

28. Prevoo ML, van 't Hof MA, Kuper HH, van Leeuwen MA, van de Putte LB, van Riel PL. Modified disease activity scores that include twenty-eight-joint counts. Development and validation in a prospective longitudinal study of patients with rheumatoid arthritis. Arthritis Rheum. 1995; 38:44-8.

29. Fransen J, van Riel PL. The Disease Activity Score and the EULAR Response Criteria. Clin Exp Rheumatol. 2005;23: S93-S99.

30. Cardiel MH, Abello-Banfi M, Ruiz-Mercado R, Alarcón-Segovia D. How to measure health status in rheumatoid arthritis in non-English speaking patients: validation of a Spanish version of the Health Assessment Questionnaire Disability Index (Spanish HAQ-Di). Clin Exp Rheumatol. 1993; 11:117-21

31. Nana A, Slater GJ, Stewart AD, Burke LM. Methodology review: using dual-energy X-ray absorptiometry (DXA) for the assessment of body composition in athletes and active people. Int $J$ Sport Nutr Exerc Metab. 2015; 25:198-215.

32. Shepherd JA, Baim S, Bilezikian JP, Schousboe JT. Executive summary of the 2013 International Society for Clinical Densitometry Position Development Conference on Body Composition. J Clin Densitom. 2013; 16:489-95.

33. Baumgartner RN, Koehler KM, Gallagher D, Romero L, Heymsfield SB, Ross RR, et al. Epidemiology of sarcopenia among the elderly in New Mexico. Am J Epidemiol. 1998; 147:755-63

34. van Riel PL. The development of the disease activity score (DAS) and the disease activity score using 28 joint counts (DAS28). Clin Exp Rheumatol. 2014;32(5 Suppl 85): S-65-74

35. Smolen JS, Breedveld FC, Burmester GR, Bykerk V, Dougados M, Emery P, et al. Treating rheumatoid arthritis to target: 2014 update of the recommendations of an international task force. Ann Rheum Dis. 2016; 75:3-15

36. Engvall IL, Tengstrand B, Brismar K, Hafström I. Infliximab therapy increases body fat mass in early rheumatoid arthritis independently of changes in disease activity and levels of leptin and adiponectin: a randomized study over 21 months. Arthritis Res Ther. 2010;12: R197.

37. Masuko K. Rheumatoid cachexia revisited: a metabolic co-morbidity in rheumatoid arthritis. Front Nutr. 2014: 24; $1: 20$.

\section{Tables}

Table 1. Clinical characteristics in Rheumatoid Arthritis

Quantitative variables are expressed in means \pm SD and cualitative variables in frequency (\%), DAS28: Disease Activity Score of 28 joints, HAQ-DI: Health Assessment Questionnaire- 


\begin{tabular}{|c|c|c|}
\hline Variables & $\mathrm{n}=277$ & Disability \\
\hline Age, years & $57.1 \pm 10.7$ & Index, \\
\hline Disease duration, years & $12.9 \pm 9.7$ & csDMARDs: \\
\hline HAQ-DI score, units & $0.57 \pm 0.5$ & Conventional \\
\hline Functional disability (HAQ-DI $\geq 0.60)$, n (\%) & $127(45.8)$ & sinthetic \\
\hline DAS28 score, units & $3.7 \pm 1.5$ & disease- \\
\hline 1. Remission $(<2.6)$; & $73(26.4)$ & modifying \\
\hline 1. Low (2.6-3.1) & $51(18.4)$ & drugs, CRP: \\
\hline 1. Moderate (3.2-5.1) & $104(37.5)$ & C-reactive \\
\hline 1. Severe disease activity (>5.1). & 49 (17.7) & $\begin{array}{l}\text { protein, ESR: } \\
\text { erythrocyte }\end{array}$ \\
\hline Corticosteroids use, n (\%) & $235(85.6)$ & sedimentation \\
\hline Corticosteroids dose, mg/day & $4.9 \pm 2.8$ & rate, TNF- \\
\hline Corticosteroids dose/ Disease duration ratio, mg/day/years & $64.9 \pm 70.9$ & alpha: tumor \\
\hline Synthetic-DMARD, $n(\%)$ & $261(94.2)$ & necrosis \\
\hline 1. Methotrexate, n (\%) & $166(60.1)$ & $\begin{array}{l}\text { factor alpha, } \\
\text { Anti-CCP: }\end{array}$ \\
\hline 1. Leflunomide, n (\%) & $102(37)$ & Anti-cyclic \\
\hline 1. Sulfasalazine, n (\%) & $89(32.2)$ & citrullined \\
\hline 1. Azathioprine, n (\%) & $45(16.3)$ & $\begin{array}{l}\text { peptide.; Anti- } \\
\text { MCV: Anti- }\end{array}$ \\
\hline 1. Chloroquine, $n$ (\%) & $44(15.9)$ & Mutated \\
\hline Treatment failure with combined csDMARDs, n (\%) & $29(10.5)$ & Citrullinated \\
\hline Laboratory variables & & Vimentin, IL- \\
\hline $\mathrm{CRP}, \mathrm{mg} / \mathrm{L}$ & $17.2 \pm 25.1$ & 6:Interleukin- \\
\hline $\mathrm{ESR}, \mathrm{mm} / \mathrm{Hr}$ & $26.4 \pm 12.5$ & 6. \\
\hline $\mathrm{RF}, \mathrm{UI} / \mathrm{mL}$ & $152.9 \pm 385.1$ & \\
\hline Anti-CCP, RU/mL $(\mathrm{n}=173)$ & $102.4 \pm 116.2$ & \\
\hline IL-6, pg/mL $(\mathrm{n}=167)$ & $22.6 \pm 63.5$ & \\
\hline
\end{tabular}




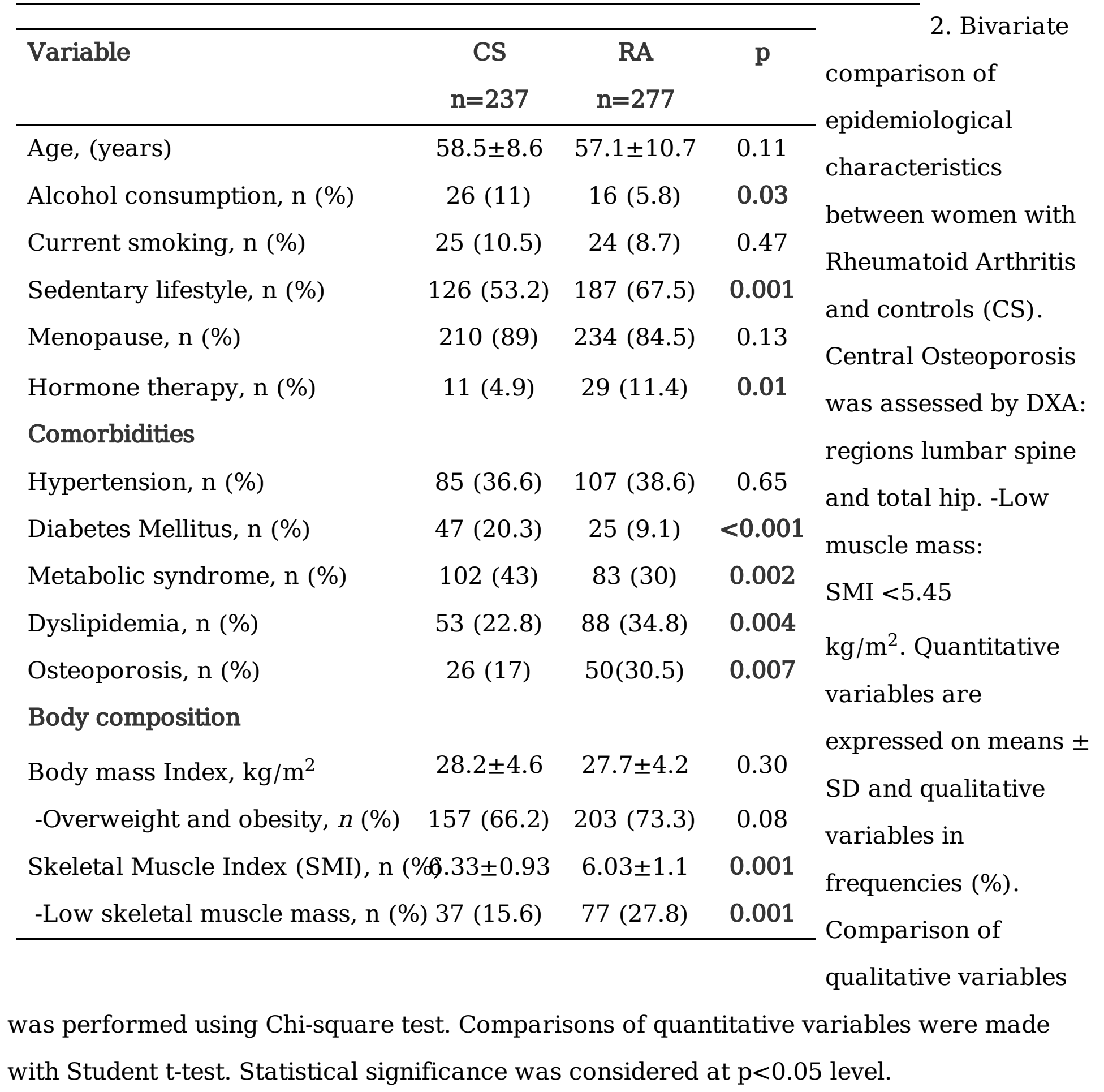

Table 3. Comparison of clinical characteristics in women with Rheumatoid Arthritis with normal vs low-skeletal muscle mass 
Weight, kg

Body mass Index, $\mathrm{kg} / \mathrm{m}^{2}$

-Overweight or obesity, n (\%)

Hypertension, n (\%)

Diabetes Mellitus, n (\%)

Clinical characteristics

Disease duration, years

HAQ-DI score, units

Functional disability, n (\%)

DAS28 score, units

Disease Activity, n (\%)

Corticosteroids use, n (\%)

Corticosteroids dose, mg/day

Synthetic DMARD, n (\%)

Treatment failure with combined

csDMARDs, n (\%)

\section{Laboratory variables}

$\mathrm{CRP}, \mathrm{mg} / \mathrm{L}$

ESR, mm/ Hr

$\mathrm{RF}, \mathrm{UI} / \mathrm{mL}$

Anti-CCP $(n=173)$

IL-6, pg/mL (n=167) $(\geq 5.45)$

$\mathrm{n}=200$

$57.5 \pm 10.4$

$11(5.5)$

18 (9)

137 (68.5)

175 (87.5)

$68.8 \pm 10.8$

$(<5.45)$

$\mathrm{n}=77$

$56.1 \pm 11.7$

0.37

5 (6.5)

0.75

$6(7.8)$

0.14

50 (64.9)

0.57

59 (76.6)

0.02

$62.2 \pm 10.8$

$<0.001$

161 (80.5)

$42(54.5)$

0.001

78 (39)

29(37.7)

0.84

$22(11)$

$3(3.9)$

0.06

$12.9 \pm 9.9$

$12.9 \pm 9.1$

0.98

$0.57 \pm 0.5$

$0.54 \pm 0.5$

0.54

93(46.7)

34 (44.7)

0.77

$3.7 \pm 1.5$

$3.7 \pm 1.4$

0.86

147(73.5)

57 (74)

0.93

170(85)

67 (87)

0.67

$6.3 \pm 7.6$

$8.3 \pm 10.1$

0.09

187(93.5)

74(96.1)

0.41

16(8)

13 (17)

0.03

$\begin{array}{ccc}14 \pm 18 & 23 \pm 36 & 0.06 \\ 26 \pm 12 & 28 \pm 14 & 0.35 \\ 116 \pm 260 & 233 \pm 565 & 0.12 \\ 101 \pm 114 & 107 \pm 124 & 0.77 \\ 22.6 \pm 66.4 & 22.6 \pm 54.7 & 0.99\end{array}$


Quantitative variables are expressed in means \pm SD and qualitative variables in frequencies (\%). Abbreviations: SMI: Skeletal Muscle Index, DAS28: Disease Activity Score of 28 joints, HAQ-DI: Health Assessment Questionnaire-Disability Index; CRP: C-reactive protein; ESR: erythrocyte sedimentation rate; TNF: tumor necrosis factor; Anti-CCP: Anti-cyclic citrullined peptide, Anti-MCV: Anti-Mutated Citrullinated Vimentin, IL-6: Interleukin-6. Comparison of qualitative variables performed with Chi-square. Comparison of quantitative variables made with Student t test. statistical significance $\mathrm{p}<0.05$

Table 4. Multivariate analysis evaluating factors associated with low-muscle mass in the total included (RA and controls). 
OR

$95 \%$ CI

p

Total included (RA + CS), n=514

Body Mass Index (BMI), $\mathrm{kg} / \mathrm{m}^{2}$

0.15

(0.09 to 0.23$)<0.001$

Rheumatoid Arthritis (RA)

2.7

(1.68 to 4.5$)$

0.03

Age (years)

alcohol consumption

Sedentary lifestyle

Menopause

Hypertension

Diabetes Mellitus

Metabolic Syndrome
Not in the model

Not in the model

Not in the model

Not in the model

Not in the model

Not in the model

Not in the model

Multivariate analysis: Logistic regression model: forward method stepwise Confidence Interval. Model 2: dependent variable: low muscle mass. Excluded covariate variables from the model: Age (years), alcohol consumption, Sedentary lifestyle, Menopause, Hypertension, Diabetes Mellitus and Metabolic Syndrome. OR, Odds Ratio; 95\% CI, 95\% statistical significance $\mathrm{p}<0.05$.

Table 5. Multivariable Logistic Regression analysis evaluating factors associated with lowmuscle mass in women with Rheumatoid Arthritis 


\begin{tabular}{lcc}
\hline OR & 95\% CI & p \\
2.3 & $(1.16$ to 4.58$)$ & 0.02 \\
2.4 & $(1.09$ to 5.37$)$ & 0.03 \\
Not in the model & - & - \\
Not in the model & - & - \\
Not in the model & - & - \\
Not in the model & - & - \\
Not in the model & - & - \\
Not in the model & - & - \\
Not in the model & - & - \\
Not in the model & &
\end{tabular}

Multivariate analysis: Logistic regression model. forward method stepwise Confidence Interval. Model with dependent variable: low skeletal muscle mass. Excluded covariate variables from the model: Age(years), Current smoking, BMI $\left(\mathrm{kg} / \mathrm{m}^{2}\right)$, Disease duration, Corticosteroids dose, Disease Activity, HAQ-DI score, Diabetes Mellitus, Metabolic Syndrome. OR, Odds Ratio; 95\% CI, 95\% statistical significance $\mathrm{p}<0.05$. 